\title{
Chronische Instabilität bei knöchernem Defekt der Schulter
}

\author{
Christian Gerhardt, Natascha Kraus, Markus Scheibel
}

\section{Zusammenfassung}

Im Zuge der voranschreitenden Entwicklung im Bereich der operativen Versorgung chronischer Schulterinstabilitäten mit knöchernen Glenoiddefekten wurden im Bereich der knöchernen Augmentationsverfahren in den letzten Jahren verschiedene arthroskopische anatomische und extraanatomische Verfahren beschrieben. Erste vielversprechende klinische und radiologische Ergebnisse nach arthroskopischem Korakoidtransfer und arthroskopischer Beckenkammspanplastik liegen vor. Auf Basis einer pathomorphologischen Klassifikation sollte die Indikationsstellung zu einer knöchernen Augmentation streng gestellt und auch knöcherne humerale, sowie weichteilige Begleitverletzungen in der Wahl der Therapie Berücksichtigung finden. Eine Überlegenheit gegenüber offenen Techniken müssen langfristige Resultate noch zeigen.

\section{Chronic Instability with Bony Defects}

In the course of an advancing development in the treatment of chronic shoulder instability with accompanying bony defects, several anatomic and extraanatomic arthroscopic techniques have recently been presented. Descriptions and first clinical and radiological results of an arthroscopic coracoid transfer, as well as J-shaped and tricortical iliac crest bone grafts have been published. On the basis of a pathomorphological classification of glenoid defects, the indication for bony augmentation should be evaluated carefully and bony humeral as well as accompanying ligamentous lesions should be taken into account when choosing treatment alternatives. Long-term results are needed to show any advantages in comparison with open techniques.

\section{Einleitung}

Die chronische Schulterinstabilität mit knöchernen Substanzverlusten der Schultergelenkspfanne stellt auch heute eine Herausforderung in der operativen Versorgung dar. In den vergangenen Jahren sind im Zuge einer voranschreitenden minimalinvasiven Chirurgie arthroskopische anatomische und extraanatomische knöcherne Augmentationsverfahren vorgestellt worden.

Dabei sollte neben der Pathomorphologie, Biologie und Biomechanik insbesondere auch das assoziierte intraartikuläre Verletzungsspektrum sowohl der knö-

OP-JOURNAL 2013; 29: 243-247

(c) Georg Thieme Verlag KG Stuttgart · New York DOI http://dx.doi.org/10.1055/s-0033-1360212

Obwohl bisher zu dieser Thematik keine biomechanischen Daten vorliegen ist davon auszugehen, dass eine Kapsel-Labrum-Rekonstruktion im Anschluss an die Spananlagerung einen zusätzlichen Stabilisierungseffekt liefert. Auch die klinischen Ergebnisse aus den offenen Knochenblockverfahren mit Versorgung der Weichteilpathologien unterstützen diese Vermutung $[16,18]$.

Über die reinen ossären Substanzverluste ohne knöchernes Fragment hinaus kann eine Indikation für eine knöcherne Augmentation auch in akuten und chronischen Fragmentdefekten des Glenoids bestehen. Eine pathomorphologische Einteilung klassifiziert diese knöchernen Defekte in 3 Typen [15]: Der Typ I besteht in akuten Glenoidfrakturen, wobei ein Typ Ia eine knöcherne Avulsionsläsion im Sinne einer knöchernen Bankart-Läsion, ein Typ Ib eine solitäre Glenoidfraktur und ein Typ Ic die akute, mehrfragmentäre Situation darstellt. In Fällen einer akuten, mehrfragmentären und nicht zu rekonstruierbaren Frakturkonfiguration kann auch hier eine knöcherne Augmentation indiziert sein. Ein Typ II kennzeichnet den chronischen Fragmentdefekt, bei welchem das verbleibende in Fehlstellung verheilte oder pseudarthrotische Fragment in seiner Größe nicht den vorherrschenden Defekt widerspiegelt. Es ist nicht bekannt, ab welchem Verhältnis von Defekt zu Fragment in der chronischen Situation ebenfalls ein knöcherner Aufbau erfolgen sollte. Womöglich sollte auch hier, bei sehr weit medial liegendem Fragment, welches an einer Artikulation nicht mehr teilnimmt, dieses keine Berücksichtigung finden und alleinig der vorhandene knöcherne Defekt der vorderen Pfanne für die Indikationsstellung ausschlaggebend sein. Chronische Erosionsdefekte (Typ III) können in einen Substanzverlust von $<25 \%$ (Typ IIIa) und $>25 \%$ (Typ IIIb) ohne vorliegendes Fragment unterteilt werden. Biomechanisch sind diese Defekte gut untersucht $[8,19]$. So ist aus
Obwohl bei knöchern bedingten Instabilitäten die Knochenblockapposition den wesentlichen Faktor in der Wiederherstellung der Stabilität der Schulter darstellt, sollten relevante Begleitpathologien adressiert werden. 
biomechanischen Arbeiten bekannt, dass ein Glenoiddefekt von $6 \mathrm{~mm}$ Länge, welches einer Breite von 25\% oder einer Länge von 19\% entspricht, die Stabilität der Schulter auch nach einem BankartRepair signifikant reduziert und hier die Indikation zu einem knöchernen Aufbau besteht [20].

Dieser knöcherne Aufbau ist arthroskopisch heute sowohl anatomisch oder extraanatomisch als auch durch intra- oder extraartikuläre Positionierung des Knochenblocks durchführbar.

Dabei bedienen sich anatomische Verfahren eines Knochenblocks aus einer anderen Körperregion als der Schulter (z.B. Beckenkammspan, distale Tibia), welcher auf Glenoidniveau zum Ausgleich des Defekts positioniert wird. Hingegen verändern extraanatomische Verfahren die Anatomie des Schultergürtels. Die Art der Spanpositionierung wird durch die relative Lage des Knochenblocks zur Gelenkkapsel definiert. Eine extraartikuläre Spanlage ist dabei durch eine Reinsertion der Kapsel am ursprünglichen Glenoid charakterisiert, sodass der intraartikuläre Teil des Glenoids identisch bleibt.

Ob hierdurch eine Normalisierung der biomechanischen Kraftverhältnisse mit einer Reduktion des Kraft/Flächen-Verhältnisses erreicht wird, ist fraglich.

\section{Arthroskopische knöcherne Augmentationsverfahren}

Die arthroskopischen Techniken der knöchernen Augmentation von anterioren Glenoiddefekten gehen auf etablierte Verfahren der offenen Chirurgie zurück.

Zwei grundlegende Techniken können hierbei unterschieden werden: der Korakoidtransfer und die knöcherne Augmentation mittels eines Beckenkammspans.

Lafosse et al. publizierten 2007 die arthroskopische Modifikation des Korakoidtransfers nach Latarjet [10] (Abb. 1). Die operative Intervention wird in Beach-Chair-Position durchgeführt. Hierzu wird nach diagnostischer Arthroskopie der Processus coracoideus präpariert. Um dies zu erreichen, muss das Rotatorenintervall reseziert werden. Nach Anlage eines korakoidalen Portals werden 2 Bohrlöcher angelegt, mit einem Führungsfaden eine Armierung vorgelegt und das Korakoid osteoto-

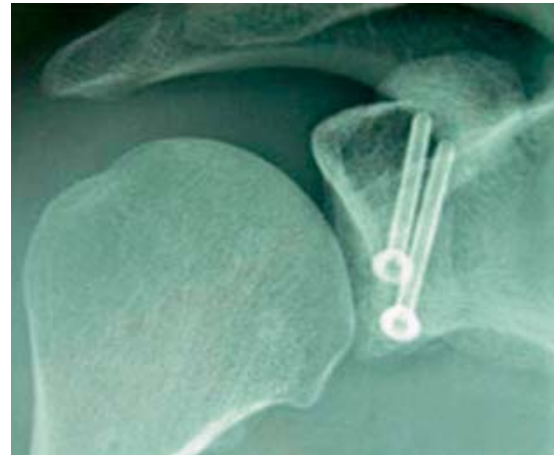

Abb. 1 Arthroskopischer Korakoidtransfer (mit freundlicher Genehmigung von Elsevier aus Lafosse L, Lejeune E, Bouchard A et al. The arthroscopic Latarjet procedure for the treatment of anterior shoulder instability. Arthroscopy 2007; 23: 1242.e1-1242.e5 [10]).

miert. Für die Positionierung des Transfers wird ein horizontaler Subscapularissplit durchgeführt und abschließend dieses mit Schrauben am vorderen Pfannenrand fixiert.

Für den offenen Korakoidtransfer sind mittlerweile zuverlässige Langzeitergebnisse vorhanden [7]. Hinsichtlich der arthroskopischen Technik wurden erste kurzfristige klinische und radiologische Ergebnisse von 12 prospektiv eingeschlossenen Patienten publiziert [1]. In dem instabilitätsspezifischen RoweScore erzielten die Patienten 92 Punkte bei einem Außenrotationsdefizit von $6^{\circ}$ im Vergleich zur gesunden Gegenseite. Im kurzen Nachuntersuchungszeitraum von 12 Monaten trat eine Reluxation auf.

\section{Arthroskopische Knochenblock- stabilisation}

\section{Extrakapsuläre Spanpositionierung}

Ein anatomisches Knochenblockverfahren mit extrakapsulärer Spanpositionierung am vorderen Glenoidhals wurde von Taverna et al. beschrieben [17] (Abb. 2).

\section{Operative Technik}

Nach Präparation des vorderen Glenoidrands und Entnahme des Beckenkammspans werden 2 Fadenanker am vorderen Glenoidrand platziert und jeweils ein Fadenschenkel nach anteroinferior und nach posterior ausgeleitet. Anschließend werden mit einem KirschnerDraht 2 Löcher im Beckenkammspan angelegt und die beiden ventralen Fadenschenkel durch diese geführt. Durch Zug an den posterioren Fäden kann nun der
Beckenkammspan über die einliegende Drehkanüle nach intraartikulär transportiert werden. Nach temporärer Fixierung mittels eines perkutan angelegten Kirschner-Drahts wird die endgültige Fixierung des Spanes mit 2 Titanschrauben, die durch ein tiefes anteroinferiores Portal eingebracht werden, durchgeführt. Abschließend wird der KapselLabrum-Komplex am originären Glenoidrand fixiert, sodass der Span letztendlich extrakapsulär zu liegen kommt.

\section{Ergebnisse}

Die Beschreibung der Technik nach Taverna et al. erfolgte am Kadavermodell, sodass zum jetzigen Zeitpunkt keine Invivo-Ergebnisse zu finden sind [17]. Es ist allerdings fraglich, ob aufgrund der Verwendung von Drehkanülen jeder Glenoiddefekt ausgleichbar ist, da der Knochenspan durch die Drehkanüle transportiert werden muss. Weiterhin sind 2 Fadenanker nur für die Platzierung des Spanes bestimmt, was zusätzliche Kosten bedeutet. Abschließend bleibt die Frage, ob der extrakapsulär positionierte Span ähnlichen biomechanischen Belastungen ausgesetzt ist wie z.B. ein intraartikulär platzierter Beckenkammspan, mit daraus potenziell veränderten Resorptionsprozessen.

\section{Intraartikuläre Spanpositionierung}

Die Techniken der arthroskopischen intraartikulären anatomischen Techniken gehen auf die Prinzipien der Knochenblockaugmentationen aus der offenen Chirurgie unter Verwendung eines J-förmigen oder trikortikalen Beckenkammspans zurück $[3,14]$.

\section{Arthroskopische J-Span-Plastik}

Die als J-Span-Plastik nach Resch bekannte Technik ist kürzlich als arthroskopisch durchführbare Variante beschrieben worden [2,3] (Abb. 3).

\section{Operative Technik}

Die Positionierung des Patienten erfolgt in Seitenlage. Nach Entnahme eines Beckenkammspans, analog zur offenen Technik mit J-förmiger Präparation, werden 2 FiberWire-Fäden durch den langen Anteil und ein K-Draht durch den kurzen Anteil des Spanes vorgelegt.

Für die arthroskopische Technik werden insgesamt 4 Portale benötigt. 
Das Einbringen des Spanes erfolgt über das tiefe anteroinferiore Portal, hierzu wird von intraartikulär ein horizontaler Split der Kapsel sowie des Subscapularis durchgeführt und die Hautinzision des tiefen anteroinferioren Portals auf ca. $2 \mathrm{~cm}$ erweitert. Ein Glenoidretraktor wird eingeführt, der beim Einbringen des Spanes als Rutsche dient. Nun wird, analog zur offenen Technik, mit einem 15-mm-Meißel etwa $5 \mathrm{~mm}$ medial des Glenoidrands ein Spalt für die Aufnahme des langen Spanschenkels geschaffen. In diesem Spalt werden 2 mit FiberWire armierte Drähte nach posterior durchgeführt und diese Fäden mit den Fäden des J-Spans verbunden. Entlang des Glenoidretraktors kann nun abschließend der Span unter Zug an den Fäden nach intraartikulär gebracht werden. Mit einem Impaktor kann der Span nun eingebolzt werden, hierbei ist der Pin, der am kurzen Anteil des Spanes eingebracht wurde, hilfreich.

\section{Ergebnisse}

Zum jetzigen Zeitpunkt existiert ein Fallbeispiel eines Patienten [3]. Ergebnisse in einer größeren Fallzahl finden sich bislang nicht. Somit ist derzeit unklar, ob diese arthroskopische Modifikation vergleichbare Resultate wie die offenen J-Span-Plastik liefert.

\section{Die arthroskopische trikortikale Beckenkammspanplastik}

In einer eigenen Erstbeschreibung wurde auch die intraartikuläre trikortikale Beckenkammspanplastik als arthroskopisches Verfahren vorgestellt [14] (Abb. 4).

\section{Operative Technik}

Ähnlich des arthroskopischen J-Spans wird der Patient in Seitenlage positioniert und der betroffene Arm über einen Armhalter leicht abduziert. Für diese Technik werden 4 Portale benötigt, das posteriore Standardportal, ein anteroinferiores Portal, ein anterosuperiores oder suprabizipitales Portal und ein tiefes anteroinferiores Portal.

Nach Anlage der Portale und Präparation des vorderen Pfannenrands erfolgt die Entnahme des trikortikalen Knochenblocks aus dem ipsilateralen Beckenkamm. Das Einbringen des trikortikalen Beckenkammspans erfolgt über das anteroinferiore Arbeitsportal. Hierzu muss die Drehkanüle entfernt werden, die

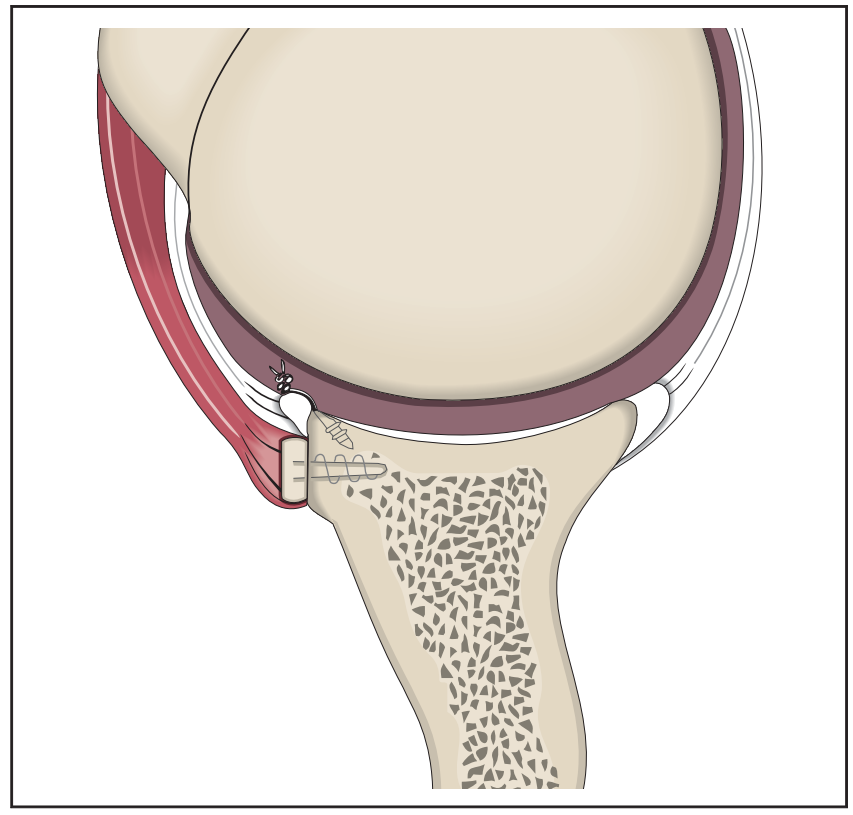

Abb. 2 Arthroskopischer extrakapsulärer Beckenkammspan.

Hautinzision um ca. $1 \mathrm{~cm}$ erweiterter werden und der Knochenblock nun mit einer geraden Fasszange eingebracht werden. Die Positionierung erfolgt zwischen Pfannenrand und Subscapularis bzw. Kapsel-Labrum-Komplex. Die Höhenpositionierung kann mit einem über das posteriore Portal eingebrachten Wechselstab sichergestellt werden. Sollte ein lateraler Überstand dennoch auftreten, kann dieser nach definitiver Fixierung des Beckenkammspans mittel Kugelfräse auf die gewünschte Höhe nivelliert werden.

Nach entsprechender Positionierung des Spanes wird über das tiefe anteroinferiore Portal der sog. Twist-Drill Guide (Arthrex, Naples, Florida) eingebracht werden. Das Charakteristikum dieser Spezialbohrhülse ist, dass sie ein doppellumiges Design aufweist, über welches ein 1,0-mm-K-Draht zur temporären Fixierung und, über das 2. Lumen, die Bohrung und das Einbringen der Schrauben erfolgen kann. Die Positionierung der Bohrhülse erfolgt nun gegen das kaudale Drittel des Spanes, wobei die K-DrahtFührungshülse nach kranial zeigen muss. Über diesen wird nun der 1,0-mm-KDraht zur temporären Fixation bikortikal eingebracht.

Ein weiterer 1,0-mm-K-Draht kann nun über das eigentliche Bohrlumen eingebracht werden und dient als Führung für den kanülierten Bohrer. Nach Gewindeschneiden kann nun die erste 3,0- 


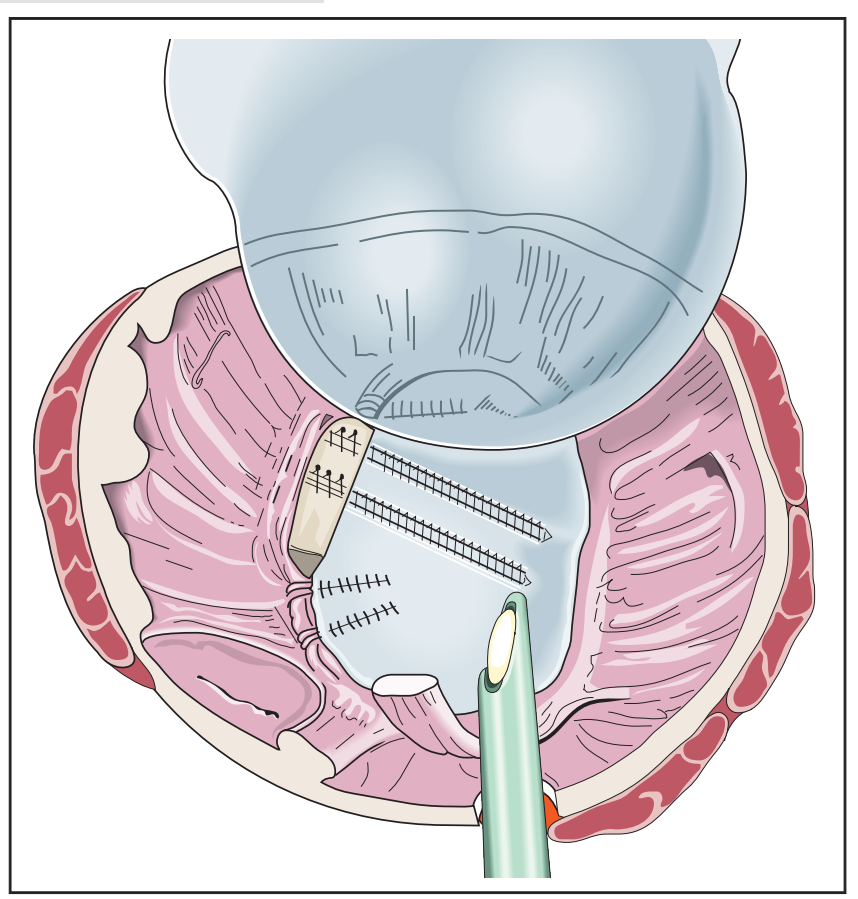

3,7 $\times 26$ mm Bio-Compression Screw (Arthrex, Naples, Forida) eingebracht werden.

Über den ersten K-Draht kann nun die Bohrhülse $180^{\circ}$ nach kranial geschwenkt und entsprechend o.g. Technik die 2. Schraube parallel zur ersten eingebracht werden (Abb.4).

Nach Anpassung der lateralen Ausdehnung des Spanes wird der Kapsel-Labrum-Komplex in PushLock-Technik am orginären Glenoid fixiert. Auf diese Art und Weise wird eine partielle Deckung der ventralen Spananteile erreicht.

\section{Ergebnisse}

Zum jetzigen Zeitpunkt wurden $15 \mathrm{~Pa}-$ tienten und 16 Schultern mit dieser arthroskopischen Beckenkammspanplastik versorgt. Entsprechend des Defektmusters wurden in 10 Patienten ein Typ-III-, in 5 Patienten ein Typ-II- und in 1 Patient ein Typ-Ic-Glenoiddefekt klassifiziert. Hinsichtlich der klinischen und radiologischen Untersuchungen erfolgte die Evaluation der Patienten prospektiv.

\section{Klinische Ergebnisse}

Nach einem durchschnittlichen Followup von 13 Monaten erzielten die Patienten im Mittel 85 Punkte im ConstantScore, 87 Punkte im Rowe-Score, 76\% im Western Ontario Shoulder Instability Index und $84 \%$ im Subjective Shoulder Value.
Abb. 4 Arthroskopische trikortikale Beckenkammspanplastik.

\section{Radiologische Ergebnisse}

In der CT-Evaluation zeigte sich präoperativ ein signifikanter Unterschied der Glenoidbreite und -fläche im Vergleich zur gesunden Gegenseite. Die unmittelbar postoperativ durchgeführte Reevaluation zeigte eine überdimensionierte Rekonstruktion mit einer Glenoidbreite von 32,4 mm bzw. und Glenoidfläche von $997 \mathrm{~mm}^{2}$. Aufgrund einer Remodellierung des Beckenkammspans zeigt sich im weiteren Verlauf eine Annäherung dieser Parameter an die Ausdehnung der gesunden Gegenseite, sodass nach 1 Jahr keine signifikanten Unterschiede gemessen werden konnten. Ähnlich verhielt es sich mit dem Glenoidindex nach Chuang et al. [4], der mit einem Wert von 1,07 nach einem Jahr keinen Unterschied zur Gegenseite aufwies.

Diese Anpassung an anatomische Werte wurde von anderen Arbeitsgruppen beobachtet und wird auf die Grundlage des Wolff'schen Gesetzes zurückgeführt.

\section{Vergleich der Techniken}

Die Apposition eines Knochenblocks ist in der Lage, die normale Gelenkbiomechanik wiederherzustellen [6].

So fanden Ghodadra et al. bei Verwendung eines auf knöchernem Glenoidniveau platzierten Beckenkammspans oder Korakoidtransfers Normalisierungen des glenoidalen Kontaktdrucks im Vergleich zur Defektsituation. Für den
Korakoidtransfer war dies bei großen Defekten nur mit der inferioren Fläche realisierbar. Ein konventionell platzierter Korakoidtransfer war nicht in der Lage, einen großen Glenoiddefekt komplett auszugleichen.

Darüber hinaus konnten Giacomo et al. eine Osteolyse von 59\% des Korakoidgrafts nach offenem Korakoidtransfer im Verlauf von 17 Monaten nachweisen [5]. Dabei war vor allem der oberflächliche und proximale Teil des Korakoids betroffen. Die Autoren schlussfolgern aus ihren Ergebnissen, dass an den anderen Teilen möglicherweise ein besserer knöcherner Kontakt, eine bessere vaskuläre Anbindung, nicht zuletzt auch durch die kurzen Beuger, und bessere biomechanische Eigenschaften durch vermehrte Belastung diese Resorption verhindern.

Auf Basis dieser Erkenntnisse muss eine andere Indikationsstellung für einen Korakoidtransfer propagiert werden, da hier nicht der Ausgleich des knöchernen Substanzdefekts im Vordergrund steht, sondern der durch die kurzen Beuger zusätzlich erreichte weichteilige Stabilisierungseffekt.

Auch nach freien Spanplastiken sind partielle Spanresorptionen beobachtet worden $[9,12]$.

Dabei zeigte sich v.a. eine Remodellierung des Spanes hin zu einer anatomischen Glenoidkonfiguration in dem Sinne, dass die Anteile des Spanes, die außerhalb der Kapsel liegen und nicht belastet sind, resorbiert werden.

So scheint eine aufwendige präoperative Defektvermessung zur genauen Spanmodellierung aufgrund dieser beobachteten Resorption nicht notwendig zu sein.

\section{Fazit}

Derzeit sind gängige offene anatomische und extraanatomische Stabilisierungsverfahren für die Schulterinstabilität mit knöchernen Glenoiddefekten in arthroskopischer Modifikation in der Literatur beschrieben worden. Teilweise liegen bereits ermutigende kurz- und mittelfristige klinische und radiologische $\mathrm{Er}$ gebnisse einiger Techniken vor. Insbesondere für die J-förmigen und trikortikalen Beckenkammspäne konnte eine anatomische Remodellierung des Glenoids beobachtet werden. Langfristige Ergebnisse müssen eine Überlegenheit 
der minimalinvasiven Techniken gegenüber klassischen offenen Verfahren noch zeigen.

\section{Literatur}

${ }^{1}$ Agneskirchner JD, Haag M, Lafosse L. [Arthroscopic coracoid transfer: indications, technique and initial results]. Orthopade 2011; 40: 41-51

${ }^{2}$ Alvik I. Tre tilfede av habituelle sculterleddsludsasjon operert a.m. Hybinette. Nord Med 1951; 45: 96

${ }^{3}$ Anderl W, Kriegleder B, Heuberer PR. All-arthroscopic implant-free iliac crest bone grafting: new technique and case report. Arthroscopy 2012; 28: 131-137

${ }^{4}$ Chuang TY, Adams CR, Burkhart SS. Use of preoperative three-dimensional computed tomography to quantify glenoid bone loss in shoulder instability. Arthroscopy 2008; 24: 376-382

${ }^{5}$ Di Giacomo G, Costantini A, de Gasperis N et al. Coracoid graft osteolysis after the Latarjet procedure for anteroinferior shoulder instability: a computed tomography scan study of twenty-six patients. J Shoulder Elbow Surg 2011; 20: 989-995

${ }^{6}$ Ghodadra N, Gupta A, Romeo AA et al. Normalization of glenohumeral articular contact pressures after Latarjet or iliac crest bonegrafting. J Bone Joint Surg Am 2010; 92 : 1478-1489

${ }^{7}$ Hovelius L, Sandstrom B, Olofsson A et al. The effect of capsular repair, bone block healing and position on the results of the Bristow-Latarjet procedure (study III): long-term follow-up in 319 shoulders. J Shoulder Elbow Surg 2012; 21: 647-660

${ }^{8}$ Itoi E, Lee SB, Berglund LJ et al. The effect of a glenoid defect on anteroinferior stability of the shoulder after Bankart repair: a cadaveric study. J Bone Joint Surg Am 2000; 82: 35-46

${ }^{9}$ Kraus N HK, Diederichs G, Scheibel M. Computertomographische Evaluation des Glenoid- remodelling nach arthroskopischer Glenoidrandrekonstruktion mittels trikortikaler Beckenkammspanplastik. In: 19. Jahreskongress der Deutschen Vereinigung für Schulter- und Ellenbogenchirurgie e.V. Berlin; 2012

10 Lafosse L, Lejeune E, Bouchard A et al. The arthroscopic Latarjet procedure for the treatment of anterior shoulder instability. Arthroscopy 2007; 23: 1242.e1-1242.e5

11 Mologne TS, Provencher MT, Menzel KA et al. Arthroscopic stabilization in patients with an inverted pear glenoid: results in patients with bone loss of the anterior glenoid. Am J Sports Med 2007; 35: 1276-1283

12 Moroder P, Hirzinger C, Lederer S et al. Restoration of anterior glenoid bone defects in posttraumatic recurrent anterior shoulder instability using the J-bone graft shows anatomic graft remodeling. Am J Sports Med 2012; 40: 1544-1550

13 Rhee YG, Cho NS. Anterior shoulder instability with humeral avulsion of the glenohumeral ligament lesion. J Shoulder Elbow Surg 2007; 16: 188-192

${ }^{14}$ Scheibel M, Kraus N. [Arthroscopic reconstruction of the glenoid concavity with an autologous bone block procedure]. Orthopade 2011; 40: 52-60

15 Scheibel M, Kraus N, Diederichs G et al. Arthroscopic reconstruction of chronic anteroinferior glenoid defect using an autologous tricortical iliac crest bone grafting technique. Arch Orthop Trauma Surg 2008; 128: 12951300

${ }^{16}$ Scheibel M, Kraus N, Gerhardt C et al. [Anterior glenoid rim defects of the shoulder]. Orthopade 2009; 38: 41-48; 50-53

17 Scheibel M, Nikulka C, Dick A et al. Autogenous bone grafting for chronic anteroinferior glenoid defects via a complete subscapularis tenotomy approach. Arch Orthop Trauma Surg 2008; 128: 1317-1325

18 Taverna E, Golano P, Pascale Vet al. An arthroscopic bone graft procedure for treating anterior-inferior glenohumeral instability. Knee Surg Sports Traumatol Arthrosc 2008; 16: 872-875
19 Warner JJ, Gill TJ, O'Hollerhan J D et al. Anatomical glenoid reconstruction for recurrent anterior glenohumeral instability with glenoid deficiency using an autogenous tricortical iliac crest bone graft. Am J Sports Med 2006; 34: 205-212

20 Yamamoto $N$, Itoi $E$, Abe $\mathrm{H}$ et al. Effect of an anterior glenoid defect on anterior shoulder stability: a cadaveric study. Am J Sports Med 2009; 37: 949-954

${ }^{21}$ Yamamoto N, Muraki T, Sperling JW et al. Stabilizing mechanism in bone-grafting of a large glenoid defect. J Bone Joint Surg Am 2010; 92: 2059-2066

22 Yiannakopoulos CK, Mataragas E, Antonogiannakis E. A comparison of the spectrum of intra-articular lesions in acute and chronic anterior shoulder instability. Arthroscopy 2007; 23: 985-990

Dr. med. Christian Gerhardt

Sektion Schulter- und

Ellenbogenchirurgie

Natascha Kraus

Sektion Schulter- und

Ellenbogenchirurgie

Univ.-Prof. Dr. med. Markus Scheibel Leitender Arzt Schulter- und Ellenbogenchirurgie

Centrum für Muskuloskeletale Chirurgie (CMSC), Campus-Mitte, Campus-Virchow Charité - Universitätsmedizin Berlin Augustenburger Platz 1

13353 Berlin

markus.scheibel@charite.de 